\title{
Patterns of cortical thinning in different subgroups of schizophrenia
}

Igor Nenadic, Rachel A. Yotter, Heinrich Sauer and Christian Gaser

\section{Background}

Alterations of cortical thickness have been shown in imaging studies of schizophrenia but it is unclear to what extent they are related to disease phenotype (including symptom profile) or other aspects such as genetic liability, disease onset and disease progression.

\section{Aims}

To test the hypothesis that cortical thinning would vary across different subgroups of patients with chronic schizophrenia, delineated according to their symptom profiles.

\section{Method}

We compared high-resolution magnetic resonance imaging data of 87 patients with DSM-IV schizophrenia with 108 controls to detect changes in cortical thickness across the entire brain $(P<0.05$, false discovery rate-adjusted). The patient group was divided into three subgroups, consisting of patients with predominantly negative, disorganised or paranoid symptoms.

\section{Results}

The negative symptoms subgroup showed the most extensive cortical thinning, whereas thinning in the other subgroups was focused in prefrontal and temporal cortical subregions.

\section{Conclusions}

Our findings support growing evidence of potential subtypes of schizophrenia that have different brain structural deficit profiles.

\section{Declaration of interest}

None.

\section{Copyright and usage}

(c) The Royal College of Psychiatrists 2015.
Analysis of cortical thinning from high-resolution magnetic resonance imaging (MRI) scans has contributed to a better understanding of cortical morphological effects of schizophrenia on the brain. Initial studies of people with mostly chronic schizophrenia demonstrated inferior and medial frontal as well as inferior and medial temporal cortical thinning ${ }^{1}$ and several subsequent studies have confirmed cortical thinning in frontal and temporal areas to varying degrees; this has included firstepisode $^{2-5}$ as well as chronic patient groups. ${ }^{6,7}$ Several subsequent imaging studies on cortical thickness have suggested that both genetic factors and those related to progressive components in the disorder might have an impact on cortical thinning. Studies in siblings or relatives of patients have produced mixed results, with some suggesting cortical thinning in temporal and/or frontal areas ${ }^{4,8-10}$ and others not supporting this finding. ${ }^{11,12}$ However, several studies have now reported associations of cortical thinning with specific schizophrenia risk genes, ${ }^{13-18}$ although effect sizes and their ultimate impact on total variability of cortical thickness remains unclear. Studies using other methods for assessment of cortical brain structure, such as voxel-based morphometry (VBM), have recently demonstrated that structural deficits might vary as a function of distinct subtypes of schizophrenia. Two large studies have demonstrated that dividing schizophrenia cohorts into three groups, such that groupings are based on dominant symptoms, reveals patterns of substantial variability in frontal and temporal subregions as well as overlap across groups. ${ }^{19,20}$ We have also recently replicated this effect of subgroup-related variability in a measure of cortical complexity. ${ }^{21}$

Regarding, cortical thinning, a recent study has shown effects related to the deficit syndrome of schizophrenia, ${ }^{22}$ but it remains unclear whether these effects are related to patterns of symptomatology, and thus possibly to a distinct phenotype, or whether it is rather related to intermediate phenotype markers of the disorder. In the present study, we tested the hypothesis that subgroups of patients with schizophrenia, delineated by their symptom profiles, would show different extents of cortical thickness changes, especially in prefrontal and temporal cortical areas. Identifying areas of overlap and divergence would highlight the role of cortical thickness as a putative intermediate phenotype. ${ }^{23}$ In order to compare cortical thickness with other markers, we used the same approach for subdividing patients with schizophrenia as used in previous VBM studies. ${ }^{19,20}$

\section{Method}

\section{Participants}

We studied a cohort of 87 patients with DSM-IV ${ }^{24}$ schizophrenia and 108 healthy controls, all of whom had provided written informed consent to a protocol approved by the Ethics Committee of the Medical School of Friedrich-Schiller-University, Jena. All participants were adults and were right-handed. ${ }^{25}$ The sample was drawn from a slightly larger cohort of 99 patients and 113 healthy controls, for which VBM analyses were presented previously, ${ }^{20}$ and identical to a sample in a recent analysis of a novel cortical complexity measure. ${ }^{21}$ Although all of the initial patients and controls passed the VBM quality control protocol, 12 participants had to be excluded based on our quality control protocol for cortical surface-based measures.

Patients were recruited from in-patient and out-patient facilities of the Jena University Hospital's Department of Psychiatry, and underwent diagnostic assessment by a psychiatrist, who also rated the severity of positive and negative symptoms on the Scale for Assessment of Positive Symptoms (SAPS) ${ }^{26}$ and Scale for Assessment of Negative Symptoms (SANS) ${ }^{26}$ respectively at the time of scanning (same week). All patients were on stable antipsychotic medication. Healthy controls had no concurrent or previous psychiatric disorder, nor major medical or neurological conditions.

Both the SANS and SAPS scores (using single items) were used to derive a delineation of three subgroups based on factor analysis with Promax rotation. Delination was based on the larger initial sample of 99 patients and 113 healthy controls. The three-subgroup 
delineation was validated in previous studies by Liddle et al. ${ }^{27,28} \mathrm{It}$ also is temporally stable, found even in older patients with chronic schizophrenia. $^{29}$

The subgroups consisted of: (a) 31 participants with predominantly negative symptoms (negative symptoms subgroup; 16 female, 15 male; mean age 35.54 years, s.d.=11.17); (b) 23 participants with predominantly disorganised symptoms (disorganised symptoms subgroup; 9 female, 14 male; mean age 35.39 years, s.d. =11.13); and (c) 33 participants with predominantly paranoid symptoms (paranoid symptoms subgroup; 14 female, 19 male; mean age 35.54 years, s.d.=11.18). These subgroups did not differ significantly from the control group (40 females, 68 males; mean age 32.16 years, s.d. $=9.99)$ in age or gender ( $t$-tests for age: control group v. negative symptoms subgroup, $P=0.135$; control group $v$. disorganised symptoms subgroup, $P=0.209$; control group $v$. paranoid symptoms subgroup, $P=0.126 ; \chi^{2}$ for gender: control group $v$. negative symptoms subgroup, $P=0.145$; control group $v$. disorganised symptoms subgroup, $P=0.852$; control group $v$. paranoid symptoms subgroup, $P=0.578$ ).

Psychopathology scores for the three subgroups, as reported previously, ${ }^{21}$ were as follows. Negative symptoms subgroup: SAPS mean 11.5 (s.d.=11.7), SANS mean 44.6 (s.d.=14.1); disorganised symptoms subgroup: SAPS mean 24.3 (s.d.=14.0), SANS mean 35.7 (s.d. $=16.5$ ); paranoid symptoms subgroup: SAPS mean 22.6 (s.d. $=18.9)$, SANS mean 27.8 (s.d. $=17.8$ ). Also, there was no difference between subgroups for disease duration (negative symptoms subgroup: mean 8.07 years, s.d. $=8.11$; disorganised symptoms subgroup: mean 10.14 years, s.d. $=7.68$; paranoid symptoms subgroup: mean 7.29 years, s.d. $=7.44$; ANOVA not significant) or age at onset (negative symptoms subgroup: mean 28.25 years, s.d. $=9.27$; disorganised symptoms subgroup: mean 26.13 years, s.d. $=11.6$; paranoid symptoms subgroup: mean 30.23 years, s.d. $=9.82$; ANOVA not significant) .

\section{Image acquisition and data analysis}

We analysed high-resolution $T_{1}$-weighted MRI scans, which were acquired for each participant on a 1.5 Tesla Phillips Gyroscan ASCII scanner (repetition time $(\mathrm{TR})=13 \mathrm{~ms}$, echo time $(\mathrm{TE})=5 \mathrm{~ms}, 256$ slices in sagittal orientation, field-of-view $256 \mathrm{~mm}$; isotropic voxel resolution: $1 \times 1 \times 1 \mathrm{~mm}^{3}$ ). Computation of cortical thickness was done using FreeSurfer software (v4.3 for Mac OS X) as described previously. ${ }^{21}$ In brief, we applied intensity normalisation, skull stripping and alignment for head position along the commissural axis, as well as labelling of cortical and subcortical structures. ${ }^{30-32}$ Quality control included visual inspection for gross imaging artefacts, as well as for poor quality of the extraction of the cortical surface. All scans included in this study used fully-automated extraction of the cortical surface with excellent results.

For statistical analysis, we tested differences in cortical thickness at three levels using separate general linear models (all implemented in a Matlab routine (Matlab 2010b), based on Statistical Parametric Mapping (SPM8) statistical routines): global thickness (across each hemisphere), regional thickness (across regions of interests (ROIs), defined by the Desikan atlas), and vertex-wise. For regional analysis, which constituted our main analysis, we applied a $P<0.05$ threshold for significance with false discovery rate (FDR) to adjust for multiple comparisons, and also included gender as a nuisance variable, since this variable might induce moderate effects even in the absence of group-level differences in sample composition. Differences were first tested between the whole patient group and the control group, and then between each of the three subgroups (negative, disorganised and paranoid symptoms) and the control group $(P<0.05$, FDR-adjusted).

\section{Results}

Patients with schizophrenia showed cortical thinning on global (hemispheric), regional and vertex-wise levels. Cortical thinning was evident across both left and right hemispheres, and was highly significant (Table 1). The effect was most pronounced for the negative symptoms subgroup.

Our main analysis of regional effects across the three subgroups (Table 2) showed that the negative symptoms subgroup showed significant cortical thinning $(P<0.05$, FDR-adjusted $)$ in prefrontal and temporal areas, as well as almost all other parietal and occipital cortical regions. The paranoid symptoms subgroup also showed significant thinning across several prefrontal and temporal areas, with sparing of the temporopolar, frontopolar and bilateral cuneus, whereas effects in inferior temporal and superior parietal areas varied across hemispheres. The disorganised symptoms subgroup showed the least cortical thinning, but still revealed several significantly thinner regions, including the superior and middle prefrontal, as well as temporal cortices.

Vertex-wise analyses showed a similar pattern, with the negative symptoms subgroup showing the most extensive deficits (Fig. 1). Regions of overlap of the three subgroups included left superior and inferior frontal, right superior temporal cortical areas.

\section{Discussion}

\section{Schizophrenia subgroups}

Before their removal in DSM-5, ${ }^{33}$ clinical subtypes of schizophrenia have been a consistent feature of diagnostic classification; however, their application in biological research has seen gradual decline. ${ }^{13,34}$ This is at odds with the widely accepted notion that the (wider) schizophrenia definition most likely encompasses several biologically distinct disease entities. Identification of putative biological markers for schizophrenia will therefore have to consider not only variability introduced by general factors (such as disease onset, disease severity and so on), but also whether this marker relates to a common deficit or to a pathophysiological feature expressed in varying degrees across subgroups.

In this study, we provide evidence that regional variability of cortical thinning in schizophrenia is related to symptom profiles and distinct subgroups. Our findings add to an increasing body

Table 1 Global (hemisphere) analysis of cortical thickness

for control and schizophrenia groups

\begin{tabular}{|c|c|c|}
\hline & $\begin{array}{c}\text { Cortical thickness, mm: } \\
\text { mean (SEM) }\end{array}$ & $P^{a}$ \\
\hline \multicolumn{3}{|l|}{ Healthy control group } \\
\hline Left hemisphere & $2.4676(0.0009)$ & \\
\hline Right hemisphere & $2.4621(0.0009)$ & \\
\hline \multicolumn{3}{|l|}{ Schizophrenia group } \\
\hline Left hemisphere & $2.3752(0.0014)$ & $1.0 \times 10^{-8}$ \\
\hline Right hemisphere & $2.3773(0.0014)$ & $2.4 \times 10^{-7}$ \\
\hline \multicolumn{3}{|c|}{ Negative symptoms subgroup } \\
\hline Left hemisphere & $2.3420(0.0040)$ & $2.0 \times 10^{-8}$ \\
\hline Right hemisphere & $2.3495(0.0040)$ & $6.5 \times 10^{-7}$ \\
\hline \multicolumn{3}{|c|}{ Disorganised symptoms subgroup } \\
\hline Left hemisphere & $2.3925(0.0045)$ & 0.001 \\
\hline Right hemisphere & $2.3911(0.0045)$ & 0.003 \\
\hline \multicolumn{3}{|c|}{ Paranoid symptoms subgroup } \\
\hline Left hemisphere & $2.3941(0.0037)$ & 0.0004 \\
\hline Right hemisphere & $2.3940(0.0038)$ & 0.002 \\
\hline
\end{tabular}




\begin{tabular}{|c|c|c|c|c|c|c|c|c|c|c|}
\hline & \multicolumn{5}{|c|}{ Left hemisphere } & \multicolumn{5}{|c|}{ Right hemisphere } \\
\hline & $\begin{array}{l}\text { Control } \\
\text { group }\end{array}$ & $\begin{array}{l}\text { Schizo- } \\
\text { phrenia } \\
\text { group }\end{array}$ & $\begin{array}{l}\text { Negative } \\
\text { symptoms } \\
\text { subgroup }\end{array}$ & $\begin{array}{l}\text { Disorganised } \\
\text { symptoms } \\
\text { subgroup }\end{array}$ & $\begin{array}{l}\text { Paranoid } \\
\text { symptoms } \\
\text { subgroup }\end{array}$ & $\begin{array}{l}\text { Control } \\
\text { group }\end{array}$ & $\begin{array}{l}\text { Schizo- } \\
\text { phrenia } \\
\text { group }\end{array}$ & $\begin{array}{l}\text { Negative } \\
\text { symptoms } \\
\text { subgroup }\end{array}$ & $\begin{array}{l}\text { Disorganised } \\
\text { symptoms } \\
\text { subgroup }\end{array}$ & $\begin{array}{l}\text { Paranoid } \\
\text { symptoms } \\
\text { subgroup }\end{array}$ \\
\hline $\begin{array}{l}\text { Banks of the superior } \\
\text { temporal sulcus }\end{array}$ & 2.611 & 2.510 & 2.461 & 2.494 & 2.567 & 2.438 & 2.324 & 2.307 & 2.313 & 2.347 \\
\hline Caudal anterior cingulate & 2.904 & 2.802 & 2.782 & 2.869 & 2.776 & 2.846 & 2.750 & 2.702 & 2.788 & 2.769 \\
\hline Caudal middle frontal & 2.680 & 2.552 & 2.526 & 2.543 & 2.584 & 2.785 & 2.706 & 2.678 & 2.710 & 2.729 \\
\hline Corpus callosum & 1.054 & 1.028 & 0.996 & 1.057 & 1.037 & 2.736 & 2.631 & 2.578 & 2.677 & 2.648 \\
\hline Cuneus & 1.953 & 1.900 & 1.876 & 1.961 & 1.881 & 2.568 & 2.461 & 2.461 & 2.464 & 2.460 \\
\hline Entorhinal & 3.219 & 3.163 & 3.080 & 3.108 & 3.279 & 3.013 & 3.023 & 3.022 & 2.939 & 3.084 \\
\hline Fusiform & 2.671 & 2.565 & 2.478 & 2.591 & 2.630 & 2.619 & 2.530 & 2.496 & 2.522 & 2.567 \\
\hline Inferior parietal & 2.558 & 2.472 & 2.435 & 2.487 & 2.497 & 2.180 & 2.113 & 2.066 & 2.159 & 2.124 \\
\hline Inferior temporal & 2.805 & 2.701 & 2.653 & 2.657 & 2.779 & 2.362 & 2.291 & 2.263 & 2.288 & 2.320 \\
\hline Isthmus cingulate & 2.842 & 2.792 & 2.720 & 2.858 & 2.813 & 2.825 & 2.723 & 2.679 & 2.742 & 2.752 \\
\hline Lateral occipital & 2.292 & 2.222 & 2.190 & 2.236 & 2.243 & 2.345 & 2.295 & 2.295 & 2.310 & 2.284 \\
\hline Lateral orbitofrontal & 2.897 & 2.778 & 2.749 & 2.757 & 2.821 & 2.898 & 2.780 & 2.748 & 2.798 & 2.797 \\
\hline Lingual & 2.151 & 2.062 & 2.011 & 2.135 & 2.058 & 2.864 & 2.758 & 2.755 & 2.740 & 2.772 \\
\hline Medial orbitofrontal & 2.519 & 2.432 & 2.382 & 2.500 & 2.430 & 3.076 & 2.959 & 2.939 & 2.974 & 2.969 \\
\hline Middle temporal & 2.892 & 2.773 & 2.729 & 2.773 & 2.815 & 2.068 & 1.986 & 1.966 & 1.983 & 2.006 \\
\hline Parahippocampal & 2.623 & 2.491 & 2.419 & 2.513 & 2.545 & 2.920 & 2.824 & 2.816 & 2.800 & 2.849 \\
\hline Paracentral & 2.433 & 2.356 & 2.314 & 2.384 & 2.376 & 2.003 & 1.941 & 1.877 & 1.958 & 1.989 \\
\hline Pars opercularis & 2.723 & 2.592 & 2.567 & 2.594 & 2.615 & 2.850 & 2.732 & 2.754 & 2.685 & 2.744 \\
\hline Pars orbitalis & 3.007 & 2.890 & 2.903 & 2.853 & 2.902 & 2.757 & 2.697 & 2.709 & 2.658 & 2.712 \\
\hline Pars triangularis & 2.673 & 2.558 & 2.561 & 2.581 & 2.538 & 2.739 & 2.642 & 2.645 & 2.642 & 2.640 \\
\hline Pericalcarine & 1.711 & 1.693 & 1.667 & 1.753 & 1.675 & 2.709 & 2.604 & 2.615 & 2.605 & 2.592 \\
\hline Postcentral & 2.094 & 2.002 & 1.971 & 2.023 & 2.017 & 2.312 & 2.247 & 2.214 & 2.279 & 2.254 \\
\hline Posterior cingulate & 2.819 & 2.738 & 2.722 & 2.778 & 2.726 & 2.708 & 2.594 & 2.553 & 2.595 & 2.633 \\
\hline Precentral & 2.444 & 2.329 & 2.300 & 2.343 & 2.346 & 2.643 & 2.553 & 2.514 & 2.577 & 2.572 \\
\hline Precuneus & 2.440 & 2.344 & 2.311 & 2.406 & 2.332 & 2.525 & 2.443 & 2.417 & 2.463 & 2.455 \\
\hline Rostral anterior cingulate & 2.868 & 2.759 & 2.717 & 2.807 & 2.765 & 2.840 & 2.682 & 2.634 & 2.700 & 2.716 \\
\hline Rostral middle frontal & 2.576 & 2.477 & 2.481 & 2.467 & 2.481 & 2.678 & 2.582 & 2.568 & 2.569 & 2.605 \\
\hline Superior frontal & 2.970 & 2.884 & 2.848 & 2.908 & 2.900 & 2.579 & 2.467 & 2.449 & 2.468 & 2.483 \\
\hline Superior parietal & 2.202 & 2.131 & 2.116 & 2.160 & 2.124 & 2.258 & 2.200 & 2.157 & 2.227 & 2.221 \\
\hline Superior temporal & 2.866 & 2.742 & 2.728 & 2.728 & 2.766 & 1.285 & 1.217 & 1.177 & 1.232 & 1.244 \\
\hline Supramarginal & 2.653 & 2.541 & 2.481 & 2.564 & 2.580 & 2.423 & 2.352 & 2.343 & 2.380 & 2.341 \\
\hline Frontal pole & 3.010 & 2.944 & 2.863 & 3.020 & 2.968 & 2.922 & 2.807 & 2.814 & 2.782 & 2.818 \\
\hline Temporal pole & 3.743 & 3.685 & 3.623 & 3.637 & 3.777 & 2.576 & 2.458 & 2.312 & 2.436 & 2.612 \\
\hline Transverse temporal & 2.382 & 2.263 & 2.223 & 2.303 & 2.272 & 0.024 & 0.037 & 0.040 & 0.035 & 0.036 \\
\hline
\end{tabular}

of research highlighting the biological heterogeneity of schizophrenia, which could provide important hints to delineating universal $v$. specific pathological pathways. ${ }^{22,35}$

Recent studies have established that regional cortical thinning is not only present at disease onset in schizophrenia, but also is evident in high-risk individuals in some areas. ${ }^{4}$ In addition, several recent studies have addressed the influence of certain clinical factors of disease and genetic risk on cortical thinning in schizophrenia. ${ }^{16,18,36}$ Yang et al have demonstrated that cortical thinning might also be observed in non-psychotic relatives of people with schizophrenia, although those with the disease had more pronounced thinning, especially in frontal and temporal areas. $^{12}$ Although this establishes both alterations related to disease liability or general phenotypic variation, there is rather little data on the heterogeneity of cortical thickness within participant groups with schizophrenia that would reflect possible subgroups within the disease entity. There is recent evidence of an association between the deficit subtype of schizophrenia and regional cortical thinning, but the results have been mixed. Whereas one found a significant difference in anterior cingulate thickness and volume, ${ }^{22}$ another study found mostly overlapping regional thinning in patients with and without deficits compared with a control group. ${ }^{35}$ Therefore, cortical thickness may be unable to separate those subtypes, albeit other morphometric markers did diverge.

Compared with the subtypes used in our study, the deficit syndrome relies mostly on outcome, i.e. longitudinal aspects. Although this might result in higher temporal stability of subgrouping, it remains to be clarified whether the deficit subtype truly correlates with a distinct biological endophenotype or merely represents a more severe disease form. Before the introduction of DSM-5, schizophrenia subtypes in DSM-III- $\mathrm{R}^{37}$ and DSM-IV ${ }^{24}$ have been based on clinical prototypes rather than biological parameters. Their validity on biological grounds has been insufficiently supported by (biological) research. ${ }^{13}$ Although our approach has also been based on psychopathology as a clinical phenotype, it has not utilised prototypes or assignment to a 


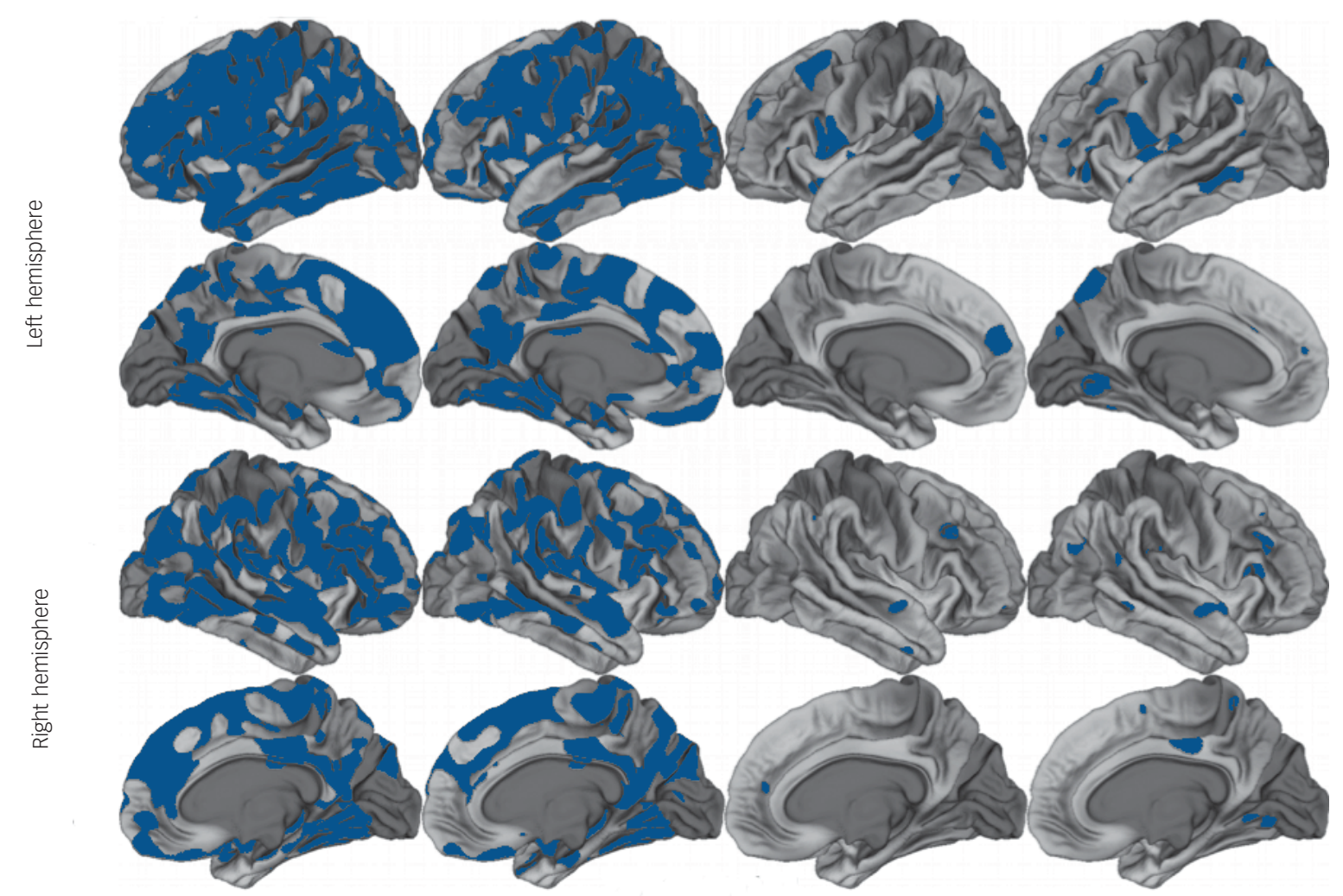

Fig. 1 Vertex-wise analysis $(P<0.05$, false discovery rate-adjusted) of cortical thickness and statistical comparison ( $v$. control group) of the entire schizophrenia group (left column), the negative symptoms subgroup (second column), disorganised symptoms subgroup (third column) and paranoid symptoms subgroup (last column).

subgroup by clinical decision, but rather has used psychometric aspects of symptom ratings to develop a more stable clinical phenotype, which is less susceptible to clinical rating biases.

\section{Differences in our findings between subgroups}

The regional patterns in frontal, temporal and cingulate areas across our three subsamples merit some attention. Our findings in the negative symptoms subgroup most closely resemble a pattern of widespread cortical thinning, almost lacking regional specificity, as seen in some recent large samples. ${ }^{6}$ Probably the greatest overlap between our three subgroups was seen in prefrontal regions, where all three groups showed convergence in areas covering both the orbitofrontal cortices (except left medial orbitofrontal cortex for the disorganised symptoms subgroup, which did not reach significance), superior and rostral middle frontal (again, except left superior frontal cortex for the disorganised symptoms subgroup) and right frontal pole regions. In contrast, temporal areas, such as the temporal pole, fusiform cortex and even superior temporal showed less convergence, as none of these areas showed thinning across all three groups. This was also the case for the medial temporal areas (parahippocampal and entorhinal). Our study did not include a detailed analysis of hippocampal thickness, which requires other analytical approaches to address the more convoluted topography. ${ }^{38}$

Considering the three subgroups in terms of how many regions showed reduction of cortical thickness, it is noteworthy that the disorganised symptoms subgroup showed the least deficits, which is consistent with recent VBM studies, where the extent of deficits was also smallest in this subgroup. ${ }^{19,20}$ Overall, the pattern of the negative symptoms subgroup showing the most widespread pathology is consistent with our previous study assessing cortical complexity, ${ }^{21}$ although cortical complexity variation (both reduction and increase) in the negative symptoms subgroup was not as widespread and extensive as the cortical thickness deficit.

\section{Limitations}

The study has some limitations. Given that this is a cross-sectional study with patients well past their first episode, the effects of disease progression, which might diverge between subgroups, ${ }^{39}$ cannot be controlled for. Subgroups might also have varied disease severity, which is difficult to assess, especially in the absence of unequivocal unidimensional markers. This is also the case for antipsychotic medication, which might at least have some subtle effects on cortical thickness (including frontal areas), although recent studies in psychosis are not suggestive of very strong effects $^{40}$ and actually demonstrate some reversibility of cortical thinning during short-term treatment. ${ }^{41}$ In participants with chronic schizophrenia, cumulative intake of antipsychotics is often difficult to assess, and given potential non-adherence to treatment, is also difficult to assess retrospectively. Chlorpromazine equivalents, which are often used in clinical studies to calculate simple dose equivalents between different antipsychotics, are likely not to be useful for this type of study, since brain structural effects of antipsychotics are probably not only related to dopamine $\mathrm{D}_{2}$ receptor occupancy or other pharmacological factors important for assessment of clinical efficacy. Finally, we also cannot rule out effects of alcohol intake or illicit substances. These effects are difficult to track, especially when assessed retrospectively.

\section{Implications}

We conclude from our findings, that although cortical thickness is reduced in schizophrenia across multiple areas, especially the prefrontal cortex, the spatial extent and effect size are variable 
across subgroups delineated by predominant symptoms. Our results warrant further exploration of the notion that different patterns of regional thinning might be related to different biological subtypes, which might then be used as different intermediate phenotypes for the dissociation of genetically distinct forms of the disease.

Igor Nenadic, MD, Department of Psychiatry and Psychotherapy, Jena University Hospital, Jena, Germany: Rachel A. Yotter, PhD, Department of Psychiatry and Psychotherapy, Jena University Hospital, Jena, Germany, and Section of Biomedical Image Analysis, University of Pennsylvania, Philadelphia, USA; Heinrich Sauer, MD, Image Analysis, University of Pennsylvania, Philadelphia, USA; Heinrich Sauer,
Department of Psychiatry and Psychotherapy, Jena University Hospital, Jena, Germany; Christian Gaser, PhD, Department of Psychiatry and Psychotherapy and Department of Neurology, Jena University Hospital, Jena, Germany

Correspondence: Igor Nenadic, Department of Psychiatry and Psychotherapy, Jena University Hospital, Philosophenweg 3, 07743 Jena, Germany. Email: igor.nenadic@uni-jena.de

First received 18 Mar 2014, final revision 4 Jun 2014, accepted 29 Sep 2014

\section{Funding}

This study was supported by grants from the Friedrich-Schiller-University of Jena (DRM 21007087 to I.N.) and the BMBF (BMBF 01EV0709 and BMBF01GW0740 to C.G.)

\section{References}

1 Kuperberg GR, Broome MR, McGuire PK, David AS, Eddy M, Ozawa F, et al. Regionally localized thinning of the cerebral cortex in schizophrenia. Arch Gen Psychiatry 2003; 60: 878-88.

2 Crespo-Facorro B, Roiz-Santianez R, Perez-Iglesias R, Rodriguez-Sanchez JM, Mata I, Tordesillas-Gutierrez D, et al. Global and regional cortical thinning in first-episode psychosis patients: relationships with clinical and cognitive features. Psychol Med 2011; 41: 1449-60.

3 Gutierrez-Galve L, Wheeler-Kingshott CA, Altmann DR, Price G, Chu EM, Leeson VC, et al. Changes in the frontotemporal cortex and cognitive correlates in first-episode psychosis. Biol Psychiatry 2010; 68: 51-60.

4 Sprooten E, Papmeyer M, Smyth AM, Vincenz D, Honold S, Conlon GA, et al. Cortical thickness in first-episode schizophrenia patients and individuals at high familial risk: A cross-sectional comparison. Schizophr Res 2013; 151 259-64.

5 Schultz CC, Koch K, Wagner G, Roebel M, Schachtzabel C, Gaser C, et al. Reduced cortical thickness in first episode schizophrenia. Schizophr Res 2010; 116: 204-9.

6 Rimol LM, Nesvag R, Hagler DJ Jr, Bergmann O, Fennema-Notestine C, Hartberg $\mathrm{CB}$, et al. Cortical volume, surface area, and thickness in schizophrenia and bipolar disorder. Biol Psychiatry 2012; 71: 552-60.

7 Palaniyappan L, Liddle PF. Dissociable morphometric differences of the inferior parietal lobule in schizophrenia. Eur Arch Psychiatry Clin Neurosci 2012; 262: 579-87.

8 Oertel-Knochel V, Knochel C, Rotarska-Jagiela A, Reinke B, Prvulovic D, Haenschel C, et al. Association between psychotic symptoms and cortical thickness reduction across the schizophrenia spectrum. Cereb Cortex 2013; 23: 61-70.

9 Byun MS, Kim JS, Jung WH, Jang JH, Choi JS, Kim SN, et al. Regional cortical thinning in subjects with high genetic loading for schizophrenia. Schizophr Res 2012; 141: 197-203.

10 Karnik-Henry MS, Wang L, Barch DM, Harms MP, Campanella C, Csernansky JG. Medial temporal lobe structure and cognition in individuals with schizophrenia and in their non-psychotic siblings. Schizophr Res 2012; 138: 128-35.

11 Boos HB, Cahn W, van Haren NE, Derks EM, Brouwer RM, Schnack HG, et al. Focal and global brain measurements in siblings of patients with schizophrenia. Schizophr Bull 2012; 38: 814-25.

12 Yang Y, Nuechterlein KH, Phillips O, Hamilton LS, Subotnik KL, Asarnow RF, et al. The contributions of disease and genetic factors towards regional cortical thinning in schizophrenia: the UCLA family study. Schizophr Res 2010; 123: 116-25.

13 Tandon R, Gaebel W, Barch DM, Bustillo J, Gur RE, Heckers S, et al. Definition and description of schizophrenia in the DSM-5. Schizophr Res 2013; 150: 3-10.

14 Walton E, Geisler D, Hass J, Liu J, Turner J, Yendiki A, et al. The impact of genome-wide supported schizophrenia risk variants in the neurogranin gene on brain structure and function. PloS One 2013; 8: e76815.

15 Blasi G, Napolitano F, Ursini G, Di Giorgio A, Caforio G, Taurisano P, et al. Association of GSK-3beta genetic variation with GSK-3beta expression, prefrontal cortical thickness, prefrontal physiology, and schizophrenia. Am J Psychiatry 2013; 170: 868-76.
16 Bergmann O, Haukvik UK, Brown AA, Rimol LM, Hartberg CB, Athanasiu L, et al. ZNF804A and cortical thickness in schizophrenia and bipolar disorder. Psychiatry Res 2013; 212: 154-7.

17 Brauns S, Gollub RL, Roffman JL, Yendiki A, Ho BC, Wassink TH, et al. DISC1 is associated with cortical thickness and neural efficiency. Neuroimage 2011; 57: 1591-600.

18 Schultz CC, Nenadic I, Koch K, Wagner G, Roebel M, Schachtzabel C, et al Reduced cortical thickness is associated with the glutamatergic regulatory gene risk variant DAOA Arg30Lys in schizophrenia. Neuropsychopharmacology 2011; 36: 1747-53.

19 Koutsouleris N, Gaser C, Jager M, Bottlender R, Frodl T, Holzinger S, et al. Structural correlates of psychopathological symptom dimensions in schizophrenia: a voxel-based morphometric study. Neuroimage 2008; 39 1600-12.

20 Nenadic I, Sauer H, Gaser C. Distinct pattern of brain structural deficits in subsyndromes of schizophrenia delineated by psychopathology. Neuroimage 2010; 49: 1153-60.

21 Nenadic I, Yotter RA, Sauer H, Gaser C. Cortical surface complexity in frontal and temporal areas varies across subgroups of schizophrenia. Hum Brain Mapp 2014; 35: 1691-9.

22 Takayanagi M, Wentz J, Takayanagi Y, Schretlen DJ, Ceyhan E, Wang L, et al. Reduced anterior cingulate gray matter volume and thickness in subjects with deficit schizophrenia. Schizophr Res 2013; 150: 484-90.

23 Nenadic I, Gaser C, Sauer H. Heterogeneity of brain structural variation and the structural imaging endophenotypes in schizophrenia. Neuropsychobiology 2012; 66: 44-9.

24 American Psychiatric Association. Diagnostic and Statistical Manual of Mental Disorders (4th edn) (DSM-IV). APA, 1994.

25 Oldfield RC. The assessment and analysis of handedness: the Edinburgh Inventory. Neuropsychologia 1971; 9: 97-113.

26 Andreasen N. Methods for assessing positive and negative symptoms. Mod Probl Pharmacopsychiatry 1990; 24: 73-88.

27 Liddle PF, Friston KJ, Frith CD, Hirsch SR, Jones T, Frackowiak RS. Patterns of cerebral blood flow in schizophrenia. Br J Psychiatry 1992; 160: $179-86$.

28 Liddle PF, Morris DL. Schizophrenic syndromes and frontal lobe performance. Br J Psychiatry 1991; 158: 340-5.

29 Sauer H, Hornstein C, Richter P, Mortimer A, Hirsch SR. Symptom dimensions in old-age schizophrenics. Relationship to neuropsychological and motor abnormalities. Schizophr Res 1999; 39: 31-8.

30 Dale AM, Fischl B, Sereno MI. Cortical surface-based analysis. I. Segmentation and surface reconstruction. Neuroimage 1999; 9: 179-94.

31 Fischl B, Sereno MI, Dale AM. Cortical surface-based analysis. II: Inflation, flattening, and a surface-based coordinate system. Neuroimage 1999; 9: 195-207.

32 Fischl B, Sereno MI, Tootell RB, Dale AM. High-resolution intersubject averaging and a coordinate system for the cortical surface. Hum Brain Mapp 1999; 8: 272-84.

33 American Psychiatric Association. Diagnostic and Statistical Manual of Mental Disorder (5th edn) (DSM-5). APA, 2013.

34 Braff DL, Ryan J, Rissling AJ, Carpenter WT. Lack of use in the literature from the last 20 years supports dropping traditional schizophrenia subtypes from DSM-5 and ICD-11. Schizophr Bull 2013; 39: 751-3.

35 Voineskos AN, Foussias G, Lerch J, Felsky D, Remington G, Rajji TK, et al. Neuroimaging evidence for the deficit subtype of schizophrenia. JAMA Psychiatry 2013; 70: 472-80.

36 Assuncao Leme IB, Gadelha A, Sato JR, Kiyomi Ota V, de Jesus Mari J, Melaragno $\mathrm{Ml}$, et al. Is there an association between cortical thickness, age of onset, and duration of illness in schizophrenia? CNS Spectrums 2013; 18: 315-21.

37 American Psychiatric Association. Diagnostic and Statistical Manual of Mental Disorders (3rd edn, revised) (DSM-III-R). APA, 1987.

38 Brambilla P, Perlini C, Rajagopalan P, Saharan P, Rambaldelli G, Bellani M, et al. Schizophrenia severity, social functioning and hippocampal neuroanatomy: three-dimensional mapping study. Br J Psychiatry 2013; 202: 50-5.

39 Nenadic I, Sauer H, Smesny S, Gaser C. Aging effects on regional brain structural changes in schizophrenia. Schizophr Bull 2012; 38: 838-44.

40 Roiz-Santianez R, Tordesillas-Gutierrez D, Ortiz-Garcia de la Foz V, Ayesa-Arriola R, Gutierrez A, Tabares-Seisdedos R, et al. Effect of antipsychotic drugs on cortical thickness. A randomized controlled one-year follow-up study of haloperidol, risperidone and olanzapine. Schizophr Res 2012; 141: 22-8.

41 Goghari VM, Smith GN, Honer WG, Kopala LC, Thornton AE, Su W, et al. Effects of eight weeks of atypical antipsychotic treatment on middle frontal thickness in drug-naive first-episode psychosis patients. Schizophr Res 2013; 149: 149-55. 